\section{Revista de CIENCIAS AMBIENTALES Tropical Journal of Environmental Sciences}

Revista de Ciencias Ambientales (Trop J Environ Sci). EISSN: 2215-3896.

(Enero-Junio, 2019) . Vol 53(1): 113-124.

DOl: http://dx.doi.org/10.15359/rca.53-1.6

Open Access: www.revistas.una.ac.cr/ambientales

EMAlL: revista.ambientales@una.ac.cr Montalvo V. H., Sáenz-Bolaños C., Cruz J. C., Carrillo E.

\title{
Amenazas y efectos potenciales del cambio climático en poblaciones silvestres de venado cola blanca (Odocoileus virginianus): Revisión de su estado de conocimiento
}

\author{
Threats and Potential Effects of Climate Change on Free Ranging Populations of \\ White-Tailed Deer (Odocoileus virginianus): A Review on the State of Knowledge
}

Víctor H. Montalvo' ${ }^{1}$ Carolina Sáenz-Bolaños ${ }^{2}$, Juan Carlos Cruz ${ }^{3}$, Eduardo Carrillo J. ${ }^{4}$

[Recibido: 18 de junio, 2018. Aceptado: 16 de octubre, 2018. Corregido: 02 de noviembre, 2018. Publicado: 1 de enero, 2019]

Nota: Resultados preliminares de este artículo fueron presentados en el I Congreso Centroamericano de Ciencias de la Tierra y el Mar, organizado por la Facultad de Ciencias de la Tierra y el Mar de la Universidad Nacional. 13-15 noviembre 2017, San José, Costa Rica.

\section{Resumen}

Las poblaciones silvestres de venado cola blanca han mostrado respuestas negativas a la variación climática dentro de su rango de distribución. En escenarios globales de cambio climático, los grupos conservacionistas deben entender las manifestaciones de la variabilidad climática en el comportamiento y distribución de poblaciones vulnerables para el desarrollo de estrategias de manejo. Este estudio tiene como objetivo identificar y sintetizar bibliografía que describa los principales efectos potenciales del cambio climático en las poblaciones de venado cola blanca a lo largo de su rango de distribución. Se realizó una intensa revisión bibliográfica mediante dos buscadores científicos en internet (Google Scholar; Web of Science) del 2000 al 2016. Durante la búsqueda se registraron documentos referentes al efecto del clima en poblaciones de venado cola blanca. Como resultado, de los 4000 documentos publicados durante el periodo evaluado (los últimos 16 años), se encontraron 18 documentos formalmente publicados en revistas peer reviewed, los cuales evidencian manifestaciones climáticas en poblaciones silvestres de venado cola blanca. Las principales respuestas observadas fueron las siguientes: cambios en la distribución, aumentos en la incidencia de enfermedades, cambios en los patrones de movimiento, cambios en la demografía poblacional, disminución de la calidad del forraje y cambios en el hábitat. Se concluye que la información disponible es limitada, restringida a la distribución norte del venado cola blanca, la cual no muestra información sobre los ecosistemas tropicales más allá de México, por lo que se necesitan, con urgencia, herramientas y enfoques para evaluar los efectos a largo y mediano plazo de la variabilidad climática en poblaciones de ungulados tropicales, pues la falta de información, al respecto, es evidente.

Palabras clave: Cambio climático; conservación; Odocoileus; ungulados; venado.

1 Investigador del Instituto Internacional en Manejo y Conservación de Vida Silvestre, Universidad Nacional, Costa Rica, victor.montalvo. guadamuz@una.cr

2 Investigadora del Instituto Internacional en Manejo y Conservación de Vida Silvestre, Universidad Nacional, Costa Rica, csaenzb@una.cr 3 Colaborador de Osa Conservation, Costa Rica, juancarloscruz@osaconservation.org

4 Investigador del Instituto Internacional en Manejo y Conservación de Vida Silvestre, Universidad Nacional, Costa Rica, eduardo.carrillo. jimenez@una.cr

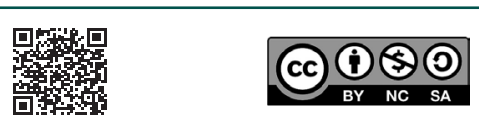

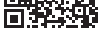

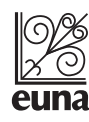




\section{Revista de CIENCIAS AMBIENTALES Tropical Journal of Environmental Sciences}

Revista de Ciencias Ambientales (Trop J Environ Sci). EISSN: 2215-3896.

(Enero-Junio, 2019) . Vol 53(1): 113-124.

Dol: http://dx.doi.org/10.15359/rca.53-1.6

Open Access: www.revistas.una.ac.cr/ambientales EMAIL: revista.ambientales@una.ac.cr Montalvo V. H., Sáenz-Bolaños C., Cruz J. C., Carrillo E.

\begin{abstract}
Free ranging populations of white-tailed deer have shown negative responses to climatic variation within their distribution. Deer populations are not categorized by the IUCN as endangered species, but climate change is still a threat to this economically and ecologically important species. This study aims to identify and synthesize literature describing the main effects of climate change on white tailed deer populations. Internet search engines were used to review literature from 2000 to 2016 in topics such as white-tailed deer and climate. As a result, 18 peer-review papers out of 4000 were found in the last 16 years, tracking climate responses on deer population. The main responses based on the literature included changes in deer distribution, increases in the incidence of diseases, changes in movement, changes in population demographics, decreasing of forage quality, and habitat changes. Finally, concluding that information available is limited, restricted to the northern distribution of white-tailed deer, and showing no information on tropical ecosystems beyond Mexico, tools and approaches to assess long-term effects of climate change on deer populations are urgently needed, specifically in places where the lack of information is evident.
\end{abstract}

Keywords: climate change; conservation; deer; Odocoileus; ungulates.

\title{
1. Introducción
}

La variabilidad climática ha incrementado repentinamente durante las últimas décadas, representando una importante preocupación para la conservación global, pues incluye potenciales efectos negativos en personas, producción alimentaria y biodiversidad (Eguiguren-Velepucha et al., 2016; Young et al., 2014). En escenarios de cambio global, conservacionistas y quienes toman decisiones deben comprender el efecto de la variabilidad climática en la abundancia, distribución y comportamiento de las poblaciones más vulnerables, para desarrollar estrategias de manejo basadas en nuevas líneas de investigación que promuevan la persistencia a largo plazo de estas especies (Dawnson et al., 2011; DeGregorioa, 2015). Proyecciones globales de temperatura estiman un incremento entre 1.5 y $3.5^{\circ} \mathrm{C}$, lo que se traduce en grandes oscilaciones y variabilidad en la media de temperatura global (IPCC 2014). Con respecto a la precipitación, las proyecciones globales apuntan a un aumento en la frecuencia de eventos extremos, muestra cambios en la distribución espaciotemporal, independientemente del escenario (Newman et al., 2011). Estos cambios abruptos en la variación climática se manifiestan en una amplia gama de respuestas y cambios en las especies (Macdonald et al., 2009; Stoner y Timm, 2011), afectan organismos y especies vulnerables que directamente representan un costo ecológico y económico a la población (Simard et al., 2010). El venado cola blanca (Odocoileus virginianus) es una especie de importancia económica a lo largo de su rango de distribución; sin embargo, los cambios en su hábitat como consecuencia del cambio climático han contribuido a la disminución y desplazamiento de sus poblaciones hacia lugares donde hostiles sequías e inviernos generan un alto gasto energético y una substancial reducción de recursos (Dawe et al., 2016; Lashey y Harper, 2012). A pesar de que organismos internacionales como la UICN (Unión Internacional para la Conservación de la Naturaleza) han descrito al venado cola blanca como una especie de menor preocupación (UICN 2018), más al sur de su distribución las poblaciones en vida libre, experimentan múltiples amenazas y un faltante de información para la toma de decisiones (Gallina y

\begin{tabular}{|c|c|c|}
\hline 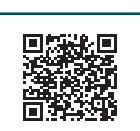 & (c) (i) (8) (2) & 114 \\
\hline
\end{tabular}




\section{Revista de CIENCIAS AMBIENTALES Tropical Journal of Environmental Sciences}

Revista de Ciencias Ambientales (Trop J Environ Sci). ElSSN: 2215-3896.

(Enero-Junio, 2019) . Vol 53(1): 113-124.

D0l: http://dx.doi.org/10.15359/rca.53-1.6

Open Access: www.revistas.una.ac.cr/ambientales

EMAlL: revista.ambientales@una.ac.cr Montalvo V. H., Sáenz-Bolaños C., Cruz J. C., Carrillo E.

Arévalo, 2016). Este esfuerzo pretende sintetizar bibliografía existente para identificar vacíos de información y ayudar a diseminar conocimientos sobre las posibles consecuencias del cambio climático en poblaciones silvestres de venado cola blanca.

\section{Metodología}

Se realizó una extensa revisión bibliográfica de las posibles consecuencias del cambio climático en poblaciones de venado cola blanca utilizando buscadores digitales de artículos científicos; Web of Science - colección de aproximadamente 12000 revistas científicas, libros y resúmenes de conferencias que cuentan con un repositorio que excede el billón de documentos, Google Scholar - base de datos de libre acceso con citaciones completas de casi todas las disciplinas incluyendo la mayoría de artículos peer-reviewed de Europa y Estados Unidos. La búsqueda web fue delimitada temporalmente del 2000 al 2016, se anotaron las siguientes combinaciones de palabras clave en el idioma inglés: "Effect of climate on Odocoileus virginianus", "Climate + Odocoileus virginianus", "Climate responses of Odocoileus virginianus", "Seasonality on white tailed deer", "Climate change + White tailed deer", "Threats of climate change on white tailed deer", Climate change + White tailed deer + tropics".

\section{Resultados y discusión}

Se encontraron 4374 artículos peer-reviewed, de los cuales únicamente 18 describieron algún efecto de la variabilidad climática en poblaciones de venado cola blanca; de estos 18 documentos consultados únicamente nueve abordaron temas directamente relacionados con cambio climático y venados cola blanca. De estos estudios solamente dos describieron manifestaciones del cambio climático en poblaciones de venado cola blanca en el trópico (Cuadro 1), lo cual resalta grandes vacíos de información relacionados con este tópico en ecosistemas tropicales (Cuadro 1).

Cuadro 1. Síntesis de respuestas eco-biológicas del efecto de la variabilidad climática en poblaciones silvestres de venado cola blanca (Odocoileus virginianus) reportadas a lo largo de su rango de distribución periodo 2000-2016

\begin{tabular}{|c|c|c|c|}
\hline Zona & País & Patrón/Respuesta & Referencia \\
\hline Templada & Canadá & Expansión del rango de acción & Lesage et al. (2000) \\
\hline Templada & Canadá & Competencia por forraje & Patterson y Power (2002) \\
\hline Templada & Canadá & Comportamiento migratorio & Sabine et al. (2002) \\
\hline Tropical & México & Cambios en movimientos & Bello et al. (2004) \\
\hline Templada & Estados Unidos de América & Cambios en movimientos & Brinkman et al. (2005) \\
\hline Templada & Estados Unidos de América & Fluctuaciones de patógenos zoonóticos & Ostfeld et al. (2006) \\
\hline Templada & Estados Unidos de América & Incremento de mortalidad & Lishawa et al. (2007) \\
\hline
\end{tabular}

\begin{tabular}{|c|c|c|c|c|c|}
\hline 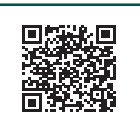 & (c) (i) () (2) & 8 & $\frac{20 \%}{20 \%}$ & 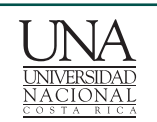 & 115 \\
\hline
\end{tabular}




\section{Revista de CIENCIAS AMBIENTALES Tropical Journal of Environmental Sciences}

Revista de Ciencias Ambientales (Trop J Environ Sci). EISSN: 2215-3896.

(Enero-Junio, 2019) . Vol 53(1): 113-124

Dol: http://dx.doi.org/10.15359/rca.53-1.6

Open Access: www.revistas.una.ac.cr/ambientales

EMAIL: revista.ambientales@una.ac.cr Montalvo V. H., Sáenz-Bolaños C., Cruz J. C., Carrillo E.

$\begin{array}{llll}\text { Templada } & \text { Estados Unidos de América } & \text { Comportamiento migratorio } & \text { Fieberg } \text { et al. (2008) } \\ \text { Templada } & \text { Estados Unidos de América } & \text { Aumento en la incidencia de patógenos } & \text { Seeleman } \text { et al. (2009) } \\ \text { Templada } & \text { Canadá } & \text { Fluctuaciones poblacionales } & \text { Simard } \text { et al. }(2010) \\ \text { Templada } & \text { Estados Unidos de América } & \text { Cambios en la calidad nutricional del forraje } & \text { Lashley y Harper (2012) } \\ \text { Templada } & \text { Estados Unidos de América } & \text { Efecto cascada en la cadena trófica } & \text { Frelich } \text { et al. }(2012) \\ \text { Templada } & \text { Canadá } & \text { Cambios en distribución } & \text { Dawe y Boutin (2014) } \\ \text { Tropical } & \text { México } & \text { Contaminación de patógenos } & \text { Estrada-Peña et al. (2014) } \\ \text { Templada } & \text { México } & \text { Aumento de la abundancia de parásitos } & \text { Feria-Arroyo et al. (2014) } \\ \text { Templada } & \text { Estados Unidos de América } & \text { Colisiones vehiculares } & \text { Olson } \text { et al. }(2015) \\ \text { Templada } & \text { Estados Unidos de América } & \text { Susceptibilidad por depredación } & \text { Hoving y Notaro (2015) } \\ \text { Templada } & \text { Canadá } & \text { Expansión del rango de distribución } & \text { Dawe y Boutin (2016) }\end{array}$

\subsection{Cambios en la distribución}

El conocimiento de la distribución de los organismos y su relación con el ambiente físico es vital para comprender el efecto del cambio climático en poblaciones silvestres (Newman, 2011). Como respuesta a las variaciones del clima, las poblaciones silvestres de ungulados han extendido su rango de distribución ocasionando un efecto cascada en otras especies, a su vez, este desplazamiento ha tenido un efecto negativo en comunidades de plantas no adaptadas al intenso forrajeo causado por medianos y grandes herbívoros (Fordham et al., 2013). Estudios recientes han sugerido el uso de modelos predictivos que describan potenciales rutas de expansión de ungulados para permitir el desarrollo de estrategias de adaptación y mitigación del cambio climático en poblaciones silvestres. Dawe y Boutin (2016) en Canadá identificaron respuestas negativas de la expansión en la distribución norte del venado cola blanca mediante el uso de modelos de distribución. Describen cómo la dinámica depredador presa potencialmente podría verse afectada, debido a mayor disponibilidad de un recurso presa para poblaciones de lobos (Canis lupus), conduciendo, así, a un incremento en poblaciones de depredadores que paralelamente generarían un aumento en las tazas de depredación de otras especies de herbívoros amenazados como el caribou (Rangifer tarandus) (Latham et al., 2011).

Por tanto, se predice que si la variabilidad climática continúa a este ritmo, es posible que las poblaciones de venado cola blanca se extiendan $100 \mathrm{~km}$ hacia el norte en los próximos 50 años (Dawe y Boutin, 2016). En lo que respecta a ambientes estacionales, Stoner y Timm (2011) han encontrado una adversa reducción en poblaciones de depredadores clave como el jaguar (Panthera onca) y puma (Puma concolor), a consecuencia de un efecto cascada debido a la disminución de poblaciones de grandes herbívoros en respuesta al estrés fisiológico ocasionado por extensas sequías e inundaciones. Finalmente, llaman la atención a la necesidad de estudios fisiológicos que permitan describir adaptaciones a nivel de individuos en relación con gradientes ambientales y ecosistémicos.

\begin{tabular}{|c|c|c|}
\hline 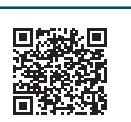 & (c) (i) (s) () () & 116 \\
\hline
\end{tabular}




\section{Revista de CIENCIAS AMBIENTALES Tropical Journal of Environmental Sciences}

Revista de Ciencias Ambientales (Trop J Environ Sci). EISSN: 2215-3896.

(Enero-Junio, 2019) . Vol 53(1): 113-124.

Dol: http://dx.doi.org/10.15359/rca.53-1.6

Open Access: www.revistas.una.ac.cr/ambientales

EMAIL: revista.ambientales@una.ac.cr Montalvo V. H., Sáenz-Bolaños C., Cruz J. C., Carrillo E.

\subsection{Cambios en la incidencia de enfermedades}

La respuesta de los patógenos a la variación climática es pobremente conocida; sin embargo, el conocimiento disponible está limitado a poblaciones de vectores y a cómo la expansión de estos vectores puede incrementar la diseminación de enfermedades (Newman, 2011). La mayoría de estudios disponibles se basan en generalizaciones simplistas que tratan de explicar cómo la incidencia de enfermedades puede fluctuar en relación con la variación climática (Altizer et al., 2013). Por ejemplo, Feria-Arroyo et al. (2014) desarrollaron modelos de distribución especialmente explícitos para describir el riesgo de diseminación de la bacteria Borrelia burgdorferi (causante de la enfermedad de Lyme) basándose en la distribución de su vector primario Ixodes scapularis (garrapata del venado).

De manera análoga para determinar la variación estacional de enfermedades zoonóticas como la enfermedad de Lyme, Ostfeld et al. (2006) analizaron el efecto combinado de la precipitación, la temperatura, abundancia del ratón de patas blancas (Peromiscus leucopus); venado cola blanca; la ardilla rayada (Tamias striatus) y la disponibilidad de bellotas (Quercus ssp) y encontraron una relación entre la incidencia de la enfermedad de Lyme asociada a los picos de abundancia de los hospederos durante estadios inmaduros del vector. Otra evidencia ha mostrado cómo las fluctuaciones climáticas pueden afectar la incidencia del reovirus Orbivirus ssp. en poblaciones de venado cola blanca; esta indica que al inicio y final de la estación seca la temperatura y la precipitación incrementan la incidencia anual de venados infectados con el reovirus (Sleeman et al., 2009).

En México, Estrada-Peña et al. (2014), mediante el uso de simulaciones y modelos, mejoraron la salud de un hato ganadero mediante el entendimiento de factores climáticos y características del paisaje que influenciaban los brotes de garrapatas en el ganado. Determinaron el mejor periodo climático y lugares clave para vacunar poblaciones silvestres de venado cola blanca, dado que es un importante hospedero de la garrapata Rhipicepahlus ssp. En lugares donde los venados coexisten con ganado vacuno, lograron exitosamente detener la diseminación de garrapatas después de años sin éxito (Estrada-Peña et al., 2014).

\subsection{Cambios en movimientos}

Los movimientos de grandes herbívoros usualmente se ven alterados por la distribución de un recurso limitante, contrayendo y expandiendo sus rangos de acción en relación con la disponibilidad de refugio, agua y alimento (Owen-Smith et al., 2005; Stoner y Timm, 2011), por lo que, a causa de variaciones climáticas como extensos inviernos y sequías, los organismos deben adaptarse a estos cambios agrestes.

En Minnesota, para venados cola blanca, se ha documentado cómo, durante los inviernos, los rangos de acción tienden a aumentar en comparación con los veranos, evidenciando una correlación positiva entre las tasas de migración y la profundidad de la nieve (Brinkman et al., 2005), patrón similar al observado en Canadá donde se notó un patrón de migración durante el

\begin{tabular}{|c|c|c|}
\hline 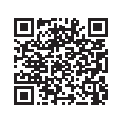 & (c) (i) (9) (2) & 117 \\
\hline
\end{tabular}




\section{Revista de CIENCIAS AMBIENTALES Tropical Journal of Environmental Sciences}

Revista de Ciencias Ambientales (Trop J Environ Sci). EISSN: 2215-3896.

(Enero-Junio, 2019) . Vol 53(1): 113-124.

Dol: http://dx.doi.org/10.15359/rca.53-1.6

Open Access: www.revistas.una.ac.cr/ambientales

EMAIL: revista.ambientales@una.ac.cr

Montalvo V. H., Sáenz-Bolaños C., Cruz J. C., Carrillo E.

verano pero con un alto nivel de filopatría (Lesage et al., 2000), resaltan el efecto de la estacionalidad como un factor que puede condicionar las distancias de una migración. Más al sur de su distribución, el principal factor que condiciona la magnitud de los movimientos del venado cola blanca son las sequias y su extensión (Lashley y Harper, 2012), sin observar diferencias estacionales en sus movimientos cuando el agua está homogéneamente accesible durante la estación seca (Webb et al., 2007). Otro comportamiento reportado en venados cola blanca en zonas áridas es la reducción de su rango de acción como estrategia para maximizar su presupuesto de energía, al disminuir la actividad de sus funciones vitales como estrategia para lidiar con la escasez de agua (Bello et al., 2004).

\subsection{Cambios nutricionales en la calidad del forraje}

La producción primaria es controlada por la precipitación y su distribución temporal, por lo tanto, la calidad y disponibilidad de nutrientes en comunidades vegetales está relacionada con la variación estacional de la precipitación (Jaramillo et al., 2011). En Quebec, Canadá, Taillon et al., (2006) encontraron como el sobre-forrajeo afectó la composición del bosque, al suprimir especies que garantizaban un alto contenido nutricional durante el invierno. Como consecuencia, durante extensos inviernos el forraje de baja calidad gradualmente redujo la condición corporal de estos individuos afectando su actividad durante el invierno. En contraste, en ambientes áridos durante periodos de sequías, la calidad del forraje disminuye también la condición corporal del venado cola blanca, por tanto, como estrategia de sobrevivencia, los venados aumentan la diversidad de especies a forrajear, debido a que pocas pueden satisfacer sus requerimientos nutricionales; optimizan, así, el presupuesto energético invertido en forrajeo (Lashley y Harper, 2012).

\subsection{Evidencia de la influencia del clima en la demografía poblacional}

La variabilidad climática es crítica para comprender parámetros como fecundidad, sobrevivencia, mortalidad, inmigración y emigración (Newman, 2011). Por ello, el estrés fisiológico asociado con extensos inviernos o veranos puede reducir la producción anual de cervatillos, así como la probabilidad de sobrevivencia y reproducción durante periodos de escases (Newman, 2011; Simard et al., 2009). Por ejemplo, estudios con caribou (Rangifer tarandus) en Groenlandia han revelado una reducción en el éxito reproductivo como consecuencia del cambio climático, que al mismo tiempo ha afectado comunidades indígenas que viven de la cacería del caribou (Vors y Boyce, 2009). Independientemente de la región ecológica, los procesos demográficos generalmente están sincronizados con picos de abundancia de recursos, incrementando las tazas de nacimientos y sobrevivencia de infantes (Stoner y Timm, 2011).

Pocos estudios han tratado de entender la demografía y dinámica del venado cola blanca y su relación con el cambio climático. Por ejemplo, en Groenlandia un estudio con caribou reveló cómo la variabilidad climática afectó las tazas reproductivas a causa de que no podían

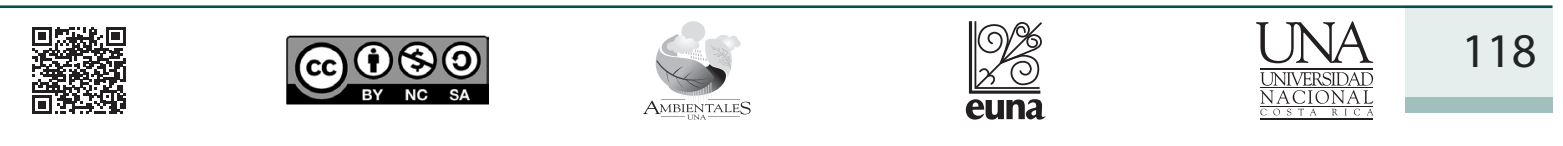




\section{Revista de CIENCIAS AMBIENTALES Tropical Journal of Environmental Sciences}

Revista de Ciencias Ambientales (Trop J Environ Sci). EISSN: 2215-3896.

(Enero-Junio, 2019) . Vol 53(1): 113-124.

D0l: http://dx.doi.org/10.15359/rca.53-1.6

Open Access: www.revistas.una.ac.cr/ambientales EMAIL: revista.ambientales@una.ac.cr Montalvo V. H., Sáenz-Bolaños C., Cruz J. C., Carrillo E.

acumular suficiente energía para llevar a cabo la reproducción y sobrevivir extensos inviernos (Vors y Boyce, 2009). Otro ejemplo en Minnesota con poblaciones de venado cola blanca reveló cómo las fluctuaciones climáticas estaban relacionadas con un aumento en la depredación por lobos, lo cual ocasionó una depresión demográfica que afectó la disponibilidad de venados para fines recreativos (Fuller, 1990). Patterson y Power (2002) también determinaron cómo el efecto acumulativo de extensos inviernos llevó a una población de venado cola blanca a un estado de desnutrición, con el consecuente aumento de la depredación por coyotes (Canis latrans) en hembras adultas y, a los pocos sobrevivientes, posteriormente, una muerte por inanición.

\subsection{Modificación del hábitat}

La modificación del hábitat como respuesta a las perturbaciones humanas es una de las amenazas más comunes para la vida silvestre (Kareiva y Marvier, 2011); sin embargo, la sinergia del cambio climático y el cambio del uso de la tierra tienen un evidente efecto en la distribución y abundancia de organismos (Boyle et al., 2013). No obstante, la variación climática ha alterado de manera negativa la dinámica temporal dentro de los hábitats, reduciendo la capacidad de carga, lo que conlleva a un declive poblacional (Stoner y Timm, 2011).

Estudios previos han evaluado el efecto del clima en las poblaciones de herbívoros, demostrando declives en las poblaciones como consecuencia de eventos climáticos que han modificado sus hábitats (Jaramillo et al., 2011; Taillon et al., 2006; Vors and Boyce, 2009), por ejemplo, en zonas templadas donde la severidad de los inviernos ha llevado a incrementar la superficie de la nieve, se dificulta la capacidad de desplazamiento de los venados cola blanca, convirtiéndolos así en una presa vulnerable ante depredadores; además del estrés fisiológico propio de la estación (Lishawa et al., 2007).

Olson et al. (2015), por su parte, determinaron que, ante eventos extremos de precipitación o nieve, el venado cola blanca tiende a utilizar las carreteras, por la dificultad de desplazarse, hecho que ocasiona un incremento en la cantidad de atropellos de venado cola blanca; resaltan, al mismo tiempo, los efectos cascada que se acumulan y llegan a crear cambios en la composición y estructura del hábitat (Frelich et al., 2012).

\section{Conclusiones}

El entendimiento del cambio climático y sus efectos negativos en poblaciones silvestres de ungulados es crítico para el diseño de estrategias de adaptación y manejo al cambio climático. Cerca de 4000 publicaciones científicas en los últimos 16 años han abordado tópicos relacionados con ecología del venado cola blanca a lo largo de su rango de distribución; no obstante, solo 18 estudios se han enfocado en entender cómo la variación climática puede afectar algún aspecto biológico de las poblaciones silvestres de venado cola blanca. A pesar de que en la distribución norte de esta especie el estado de conocimiento en poblaciones silvestres es

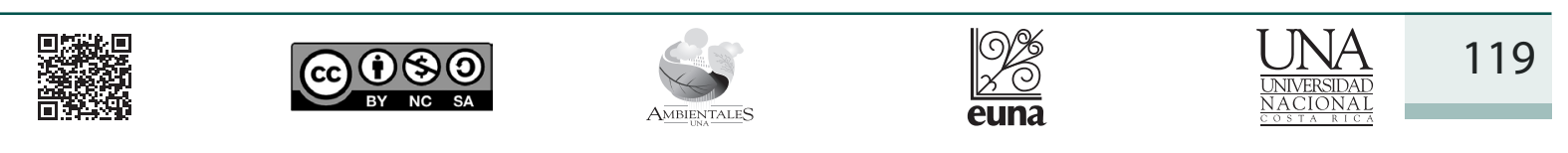




\section{Revista de CIENCIAS AMBIENTALES Tropical Journal of Environmental Sciences}

Revista de Ciencias Ambientales (Trop J Environ Sci). EISSN: 2215-3896.

(Enero-Junio, 2019) . Vol 53(1): 113-124.

DOl: http://dx.doi.org/10.15359/rca.53-1.6

Open Access: www.revistas.una.ac.cr/ambientales

EMAIL: revista.ambientales@una.ac.cr Montalvo V. H., Sáenz-Bolaños C., Cruz J. C., Carrillo E.

considerable, todavía existen grandes vacíos de información sobre las potenciales respuestas al cambio climático.

En cuanto a la distribución sur del venado cola blanca y en ambientes tropicales, la disponibilidad de información es mínima, posiblemente debido a que en Centroamérica el manejo del venado cola blanca como especie cinegética es muy restringido, además de no representar una especie con atractivo para su conservación. A través de la síntesis de bibliografía analizada del 2000 al 2016 , los pocos estudios disponibles enfatizaron en 6 manifestaciones de la variación climática: 1) cambios en la distribución de venado; 2) incremento en la incidencia de enfermedades que afectan la salud del venado cola blanca; 3 ) cambios abruptos en los movimientos y comportamiento; 4) cambios en la demografía poblacional; 5) reducción de la calidad de forraje debido a inviernos y veranos extremos; 6) cambios en la estructura y composición del hábitat como efecto cascada.

El cambio climático representa un nuevo reto para manejadores y científicos que estudian el venado cola blanca y otros ungulados, por lo que hoy en día se requiere de nuevas herramientas y enfoques para el desarrollo de iniciativas que, a largo plazo, puedan describir patrones tempranos de los efectos del cambio climático en poblaciones silvestres, especialmente en regiones tropicales como Centroamérica y Sur-América, donde la falta de información es evidente.

Si bien es cierto, el venado cola blanca es una especie de menor preocupación para la comunidad de conservación; sin embargo, su sobreabundancia o ausencia en los ecosistemas tropicales puede traer consigo efectos negativos en especies y organismos en todos los niveles de la cadena trófica. Por ello, el entendimiento de la relación entre la variabilidad climática y aspectos biológicos del venado cola blanca como especie indicadora pueden contribuir a mejorar el entendimiento de este fenómeno, además de sustentar la toma de decisiones de manejo dentro y fuera de áreas protegidas.

\section{Agradecimientos}

El especial agradecimiento al personal bibliotecario de la Universidad de Massachusetts Amherst y la Universidad Nacional, Costa Rica, por colaborar en la búsqueda de la bibliografía de difícil acceso, así como otros documentos clave para la realización de este trabajo. También, un agradecimiento a la Facultad de Tierra y Mar de la Universidad Nacional, por financiar la participación de este equipo de investigación en el I Congreso Centroamericano de Ciencias de la Tierra, con una versión preliminar de este estudio. Finalmente, agrademos a la Revista y a las personas revisoras anónimas por sus oportunos comentarios.

\section{Referencias}

Altizer, S., Ostfeld, R. S., Johnson, P. T., Kutz, S., \& Harvell, C. D. (2013). Climate change and infectious diseases: from evidence to a predictive framework. Science, 341(6145), 514-519. https://doi.org/10.1126/science.1239401

\begin{tabular}{|c|c|c|}
\hline 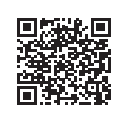 & (c) (i) $\$($ & 120 \\
\hline
\end{tabular}




\section{Revista de CIENCIAS AMBIENTALES Tropical Journal of Environmental Sciences}

Revista de Ciencias Ambientales (Trop J Environ Sci). EISSN: 2215-3896. (Enero-Junio, 2019) . Vol 53(1): 113-124. Dol: http://dx.doi.org/10.15359/rca.53-1.6 Open Access: www.revistas.una.ac.cr/ambientales EMAIL: revista.ambientales@una.ac.cr Montalvo V. H., Sáenz-Bolaños C., Cruz J. C., Carrillo E.

Bello, J., Gallina, S., \& Equihua, M. (2004). Movements of the white-tailed deer and their relationship with precipitation in northeastern Mexico. InterCiencia-Caracas, 29(7), 357-361.

Brinkman, T. J., Deperno, C. S., Jenks, J. A., Haroldson, B. S., \& Osborn, R. G. (2005). Movement of female white-tailed deer: effects of climate and intensive row-crop agriculture. Journal of Wildlife Management, 69(3), 1099-1111. https://doi.org/10.2193/0022-54 1X(2005)069[1099:MOFWDE]2.0.CO;2

Boyle M., Knopff K., Northrup j., Pitt J. \& Vors L. (2013). Harvest models for changing enviroments. In J. F.Brodie, E. S. Post, \& D. F. Doak (Eds.). Wildlife conservation in a changing climate, 293-306. Chicago.

Dawe, K. L., \& Boutin, S. (2016). Climate change is the primary driver of white tailed deer (Odocoileus virginianus) range expansion at the northern extent of its range; land use is secondary. Ecology and Evolution, 6(18), 6435-6451. https://doi.org/10.1002/ece3.2316

Dawe, K. L., Bayne, E. M., \& Boutin, S. (2014). Influence of climate and human land use on the distribution of white-tailed deer (Odocoileus virginianus) in the western boreal forest. Canadian Journal of Zoology, 92(4), 353-363. https://doi.org/10.1139/cjz-2013-0262

Dawson, T. P., Jackson, S. T., House, J. I., Prentice, I. C., \& Mace, G. M. (2011). Beyond predictions: biodiversity conservation in a changing climate. Science, 332(6025), 53-58. https://doi. org/10.1126/science.1200303

DeGregorio, B. A., Westervelt, J. D., Weatherhead, P. J., \& Sperry, J. H. (2015). Indirect effect of climate change: Shifts in ratsnake behavior alter intensity and timing of avian nest predation. Ecological Modelling, 312, 239-246. https://doi.org/10.1016/j.ecolmodel.2015.05.031

Eguiguren-Velepucha P., Chamba J., Aguirre N., Ojeda-Luna, T., Samaniego-Rojas, Furniss M., Howe C \& Aguirre Z. (2016). Tropical ecosystems vulnerability to climate change in southern Ecuador. Tropical Conservation Science, 9(4), 1-17.https://doi.org/10.1177/1940082916668007

Estrada-Peña, A., Carreón, D., Almazán, C., \& de la Fuente, J. (2014). Modeling the impact of climate and landscape on the efficacy of white tailed deer vaccination for cattle tick control in northeastern Mexico. PloS one, 9(7), e102905. https://doi.org/10.1371/journal.pone.0102905

Estrada-Peña, A., Ostfeld, R. S., Peterson, A. T., Poulin, R., \& de la Fuente, J. (2014). Effects of environmental change on zoonotic disease risk: an ecological primer. Trends in Parasitology, 30(4), 205-214. https://doi.org/10.1016/j.pt.2014.02.003

Feria-Arroyo, T. P., Castro-Arellano, I., Gordillo-Pérez, G., Cavazos, A. L., Vargas-Sandoval, M., Grover, A. \& Esteve-Gassent, M. D. (2014). Implications of climate change on the distribution of the tick vector Ixodes scapularis and risk for Lyme disease in the Texas-Mexico transboundary region. Parasites \& vectors, 7(1), 1. https://doi.org/10.1186/1756-3305-7-199

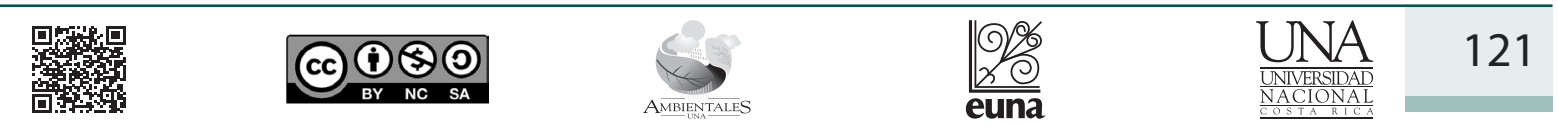




\section{Revista de CIENCIAS AMBIENTALES Tropical Journal of Environmental Sciences}

Revista de Ciencias Ambientales (Trop J Environ Sci). EISSN: 2215-3896.

(Enero-Junio, 2019) . Vol 53(1): 113-124.

D0l: http://dx.doi.org/10.15359/rca.53-1.6

Open Access: www.revistas.una.ac.cr/ambientales

EMAIL: revista.ambientales@una.ac.cr Montalvo V. H., Sáenz-Bolaños C., Cruz J. C., Carrillo E.

Fieberg, J., Kuehn, D. W., \& DelGiudice, G. D. (2008). Understanding variation in autumn migration of northern white-tailed deer by long-term study. Journal of Mammalogy, 89(6), 1529-1539. https://doi.org/10.1644/07-MAMM-A-277.1

Fordham, D. A., Akçakaya, H. R., Araújo, M. B., Keith, D. A., \& Brook, B. W. (2013). Tools for integrating range change, extinction risk and climate change information into conservation management. Ecography, 36(9), 956-964. https://doi.org/10.1111/j.1600-0587.2013.00147.x

Frelich, L. E., Peterson, R. O., Dovčiak, M., Reich, P. B., Vucetich, J. A., \& Eisenhauer, N. (2012). Trophic cascades, invasive species and body-size hierarchies interactively modulate climate change responses of ecotonal temperate-boreal forest. Philosophical Transactions of the Royal Society of London B: Biological Sciences, 367(1605), 2955-2961. https://doi.org/10.1098/ rstb.2012.0235

Fuller, T. K. (1990). Dynamics of a declining white-tailed deer population in north-central Minnesota. Wildlife Monographs, 3-37.

Gallina, S. and López Arévalo, H. (2016). Odocoileus virginianus. The IUCN Red List of Threatened Species 2016: e.T42394A22162580. Downloaded on 21 November 2016.

Garroway, C. J., \& Broders, H. G. (2005). The quantitative effects of population density and winter weather on the body condition of white-tailed deer (Odocoileus virginianus) in Nova Scotia, Canada. Canadian Journal of Zoology, 83(9), 1246-1256. https://doi.org/10.1139/z05-118

Hoving, C. L., \& Notaro, M. (2015). Ice, Snow, and Swamp: Managing Deer in Michigan's Changing Climate. Michigan Journal of Sustainability, 3. DOI: http://dx.doi.org/10.3998/ mjs.12333712.0003.008

Intergovernmental Plan on Climate Change [IPCC]. (2014). Climate Change 2014: Synthesis Report. Contribution of Working Groups I, II and III to the Fifth Assessment Report of the Intergovernmental Panel on Climate Change [Core Writing Team, R.K. Pachauri and L.A. Meyer (eds.)]. IPCC, Geneva, Switzerland, $151 \mathrm{pp}$. Recuperado de https://www.weadapt. org/sites/weadapt.org/files/legacy-new/knowledge-base/files/5554becc27072ar5-syr-final-all-topics.pdf

Jaramillo, V. J., Martínez-Yrízar, A., \& Sanford Jr. (2011). Primary productivity and biogeochemistry of seasonally dry tropical forests. In Dirzo R., Young H. S., Mooney H. A., \& G. Ceballos (Eds.), Seasonally dry tropical forests (pp. 109-128). Washington D.C. https://doi. org/10.5822/978-1-61091-021-7_7

Kareiva, P. M. M. (2011). Conservation science balancing the needs of people and nature. Greenwood Village.

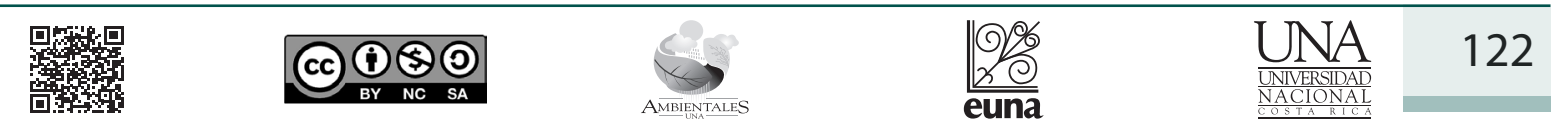




\section{Revista de CIENCIAS AMBIENTALES Tropical Journal of Environmental Sciences}

Revista de Ciencias Ambientales (Trop J Environ Sci). EISSN: 2215-3896.

(Enero-Junio, 2019) . Vol 53(1): 113-124.

Dol: http://dx.doi.org/10.15359/rca.53-1.6

Open Access: www.revistas.una.ac.cr/ambientales

EMAIL: revista.ambientales@una.ac.cr Montalvo V. H., Sáenz-Bolaños C., Cruz J. C., Carrillo E.

Lashley, M. A., \& Harper, C. A. (2012). The effects of extreme drought on native forage nutritional quality and white-tailed deer diet selection. Southeastern Naturalist, 11(4), 699-710. https://doi.org/10.1656/058.011.0409

Latham, A. D. M., Latham, M. C., McCutchen, N. A., \& Boutin, S. (2011). Invading white-tailed deer change wolf-caribou dynamics in northeastern Alberta. The Journal of Wildlife Management, 75(1), 204-212. https://doi.org/10.1002/jwmg.28

Lesage, L., Crête, M., Huot, J., Dumont, A., \& Ouellet, J. P. (2000). Seasonal home range size and philopatry in two northern white-tailed deer populations. Canadian Journal of Zoology, 78(11), 1930-1940. https://doi.org/10.1139/z00-117

Lishawa, S. C., Bergdahl, D. R., \& Costa, S. D. (2007). Winter conditions in eastern hemlock and mixed-hardwood deer wintering areas of Vermont. Canadian Journal of Forest Research, 37(3), 697-703. https://doi.org/10.1139/X06-256

McDonald, J., Christensen, S., Deblinger, R. and Woytek, W., (2009). An alternative to climate change for explaining species loss in Thoreau's woods. Proceedings of the National Academy of Sciences, pp.pnas-0811954106. https://doi.org/10.1073/pnas.0811954106

McDonald, J., Christensen, S., Deblinger, R., \& Woytek, W. (2009). An alternative to climate change for explaining species loss in Thoreau's woods. Proceedings of the National Academy of Sciences, pnas-0811954106. https://doi.org/10.1073/pnas.0811954106

Newman, J. A. (2011). Climate change biology. Massachusetts, CABI. https://doi. org/10.1079/9781845937485.0000

Ogutu, J. O., Piepho, H. P., \& Dublin, H. T. (2015). Reproductive seasonality in African ungulates in relation to rainfall. Wildlife Research, 41(4), 323-342. https://doi.org/10.1071/WR13211

Olson, D. D., Bissonette, J. A., Cramer, P. C., Bunnell, K. D., Coster, D. C., \& Jackson, P. J. (2015). How does variation in winter weather affect deer-vehicle collision rates. Wildlife Biology, 21(2), 80-87. https://doi.org/10.2981/wlb.00043

Ostfeld, R. S., Canham, C. D., Oggenfuss, K., Winchcombe, R. J., \& Keesing, F. (2006). Climate, deer, rodents, and acorns as determinants of variation in Lyme-disease risk. PLoS, 4(6), e145. https://doi.org/10.1371/journal.pbio.0040145

Owen-Smith N., Mason R., \& J. Ogutu. (2005). Correlates of survival rates for 10 African ungulate populations: density, rainfall and predation. Journal of Animal Ecology, 74(4), 774-788. https://doi.org/10.1111/j.1365-2656.2005.00974.x

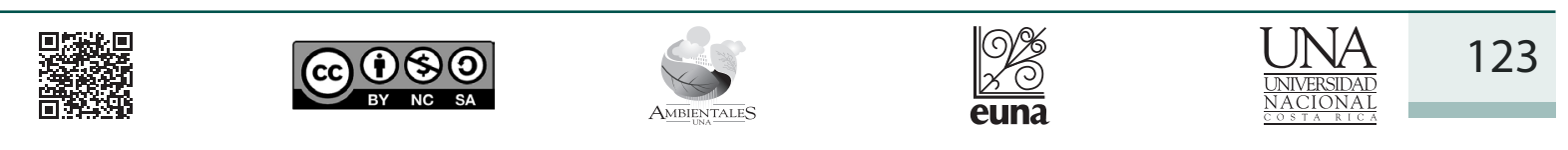




\section{Revista de CIENCIAS AMBIENTALES Tropical Journal of Environmental Sciences}

Revista de Ciencias Ambientales (Trop J Environ Sci). EISSN: 2215-3896.

(Enero-Junio, 2019) . Vol 53(1): 113-124. Dol: http://dx.doi.org/10.15359/rca.53-1.6 Open Access: www.revistas.una.ac.cr/ambientales EMAIL: revista.ambientales@una.ac.cr Montalvo V. H., Sáenz-Bolaños C., Cruz J. C., Carrillo E.

Patterson, B. R., \& Power, V. A. (2002). Contributions of forage competition, harvest, and climate fluctuation to changes in population growth of northern white-tailed deer. Oecologia, 130(1), 62-71. https://doi.org/10.1007/s004420100783

Sabine, D. L., Morrison, S. F., Whitlaw, H. A., Ballard, W. B., Forbes, G. J., \& Bowman, J. (2002). Migration behavior of white-tailed deer under varying winter climate regimes in New Brunswick. The Journal of Wildlife Management, 718-728. https://doi.org/10.2307/3803137

Simard, M. A., Coulson, T., Gingras, A., \& Côté, S. D. (2010). Influence of density and climate on population dynamics of a large herbivore under harsh environmental conditions. The Journal of Wildlife Management, 74(8), 1671-1685. https://doi.org/10.2193/2009-258

Sleeman, J. M., Howell, J. E., Knox, W. M., \& Stenger, P. J. (2009). Incidence of hemorrhagic disease in white-tailed deer is associated with winter and summer climatic conditions. EcoHealth, 6(1), 11-15. https://doi.org/10.1007/s10393-009-0220-6

Stoner, K. E., \& Timm, R. M. (2011). Seasonally dry tropical forest mammals: Adaptations and seasonal patterns. In R. Dirzo, H, S. Young, H. A. Mooney, \& G. Ceballos (Eds.), Seasonally dry tropical forests (pp. 85-106). Washington D.C. https://doi.org/10.5822/978-1-61091-021-7_6

Taillon, J., Sauvé, D. G., \& Côté, S. D. (2006). The Effects of Decreasing Winter Diet Quality on Foraging Behavior and Life-History Traits of White-Tailed Deer Fawns. The Journal of Wildlife Management, 1445-1454. https://doi.org/10.2193/0022-541X(2006)70[1445:TEODWD]2.0.CO;2

Vors, L. S., \& Boyce, M. S. (2009). Global declines of caribou and reindeer. Global Change Biology, 15(11), 2626-2633. https://doi.org/10.1111/j.1365-2486.2009.01974.x

Webb, S. L., Hewitt, D. G., \& Hellickson, M. W. (2007). Scale of Management for Mature Male White Tailed Deer as Influenced by Home Range and Movements. The Journal of Wildlife Management, 71(5), 1507-1512. https://doi.org/10.2193/2006-300

Young B., Dubois N. \& Rowland E. (2014). Using the climate change vulnerability index to inform adaptation planning: Lessons, innovations, and next steps: Using the Climate Change Vulnerability Index. Wildlife Society Bulletin, 39(1), 1-8.

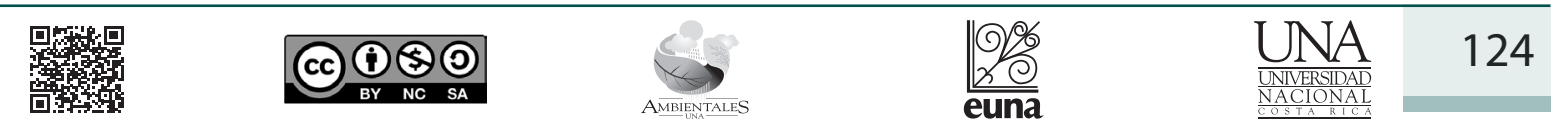

